\title{
The Jupiter-Comet Collision: some conceptual implications
}

\author{
By Dileep V. Sathe \\ Dadawala Jr. College, 1433 Kasba Peth, Pune - 411011 India
}

\section{INTRODUCTION}

A typical science course at the high school level includes some information on planets and their moons. For example, it is well-known that Jupiter has 16 moons and Saturn has 18 moons. Add to this the enthusiasm of the public in the collision of comet Shoemaker-Levy 9 with Jupiter in July 1994. This immediately raises the possibility of a collision of a comet with a moon of Jupiter. Due to this possibility a strange fact about these moons comes into the picture, that is some of them are prograde in nature and some are retrograde. Can these two types of moons pose any problems in teaching ? The present situation in education leads us to believe that they can pose some problems. It is described below, in support of this answer.

Educators from many countries have observed that the Aristotelian ideas continue to persist among graduates, in spite of learning Newtonian mechanics in colleges also. This is evident, for example, in the fact that many students think that a tangential force acts on a body performing circular motion, instead of the centripetal force. So the greatest and global problem is how to get rid of the tangential force from the minds of students and how to impregnate the centripetal force instead.

According to the present author (Sathe, 1993) a majority of students will continue to ignore the centripetal force because:

i) the concept of work and the law of parallelogram of forces strengthen the idea that there has to be a force in the direction of motion and,

ii) our inability to decide the direction of motion on the basis of force, anticlockwise or clockwise, although we do assume that the gravitational force provides the centripetal force for the planetary motion. The second reason has been termed as the $A / C$ paradox and it makes comprehension of Bohr's model of the hydrogen atom also very difficult where the electrostatic force provides the centripetal force for the electron's motion around the proton.

As prograde and retrograde moons of Jupiter are now prominently entering into discussions, due to the collision of 1994, the necessity of accepting the A/C paradox as one of the obstacles in the comprehension of basic concepts has increased considerably. The present communication considers the relevant findings from the past and suggests new directions for the future.

\section{Why the JPR System is a Challenging one?}

To understand the challenge posed by the system of Jupiter, one prograde moon and one retrograde moon - i.e. the JPR system - let us compare it with the system of Earth and Moon - i.e. the EM system.

In the EM system, one can easily get the equation of velocity of moon, assuming that the gravitational force provides the centripetal force. No problem of direction arises in this case. But in the JPR system, this assumption is not sufficient because it is necessary to explain why the prograde moon is orbiting anticlock-wise and the retrograde moon is orbiting clockwise, on the basis of gravitational or centripetal force. Therefore the JPR system is much more challenging than the EM system.

It is to be noted specifically that the use of initial conditions cannot solve this problem in the learning of basic concepts. The persistence of global misunderstanding, mentioned in the introduction, provides the validity of the foregoing statement. Hence the present author (Sathe, 1995) has appealed for the use of the JPR system instead of the EM system.

It should be noted categorically that the present treatment of uniform circular motion puts a 
lot of emphasis on the action of centripetal force. This is evident not only in textbooks but even in the questionnaires of educators. Educators always ask students to show some force acting on the body in circular motion. Therefore educators have to realize that students do expect that one should be able to decide the direction of motion anticlockwise or clockwise on the basis of force. Unfortunately, the present treatment can not fulfill this expectation.

\section{Similarity between Kepler and Students}

Lastly, it is to be noted that Kepler also has made an attempt to explain the planetary motion on the basis of sweeping action of lines of force, (Ebison, 1980). This is similar to the action of tangential force, usually thought of by even graduate students. Of course, in physics, Kepler's reasoning has taken the back seat, in view of Newton's monumental work. Nevertheless, educators of physics and astronomy also have to consider this similarity earnestly between the thinking of Kepler and students. Mathematical accuracy in physics cannot solve conceptual and educational problems.

\section{REFERENCES}

Ebison, M.G., 1980, Proceedings of the Intl. Conf, on Post Graduate Education of Physicists, Prague, Czechoslovakia, p. 160.

Sathe, D,Y., 1993, Bulletin 7 for Teaching Astronomy in the Asian-Pacific Region, p. 82.

SATHE, D.V., 1995, Physics Education, 30,327. 